\title{
ON THE RELATIONSHIP OF UC H II REGIONS AND CLASS II METHANOL MASERS. I. SOURCE CATALOGS
}

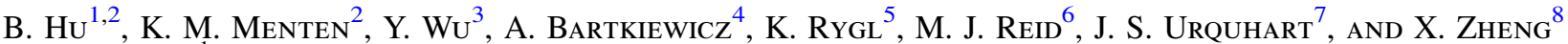 \\ ${ }^{1}$ Purple Mountain Observatory, Chinese Academy of Science, Nanjing 210008, China; hubonju@ gmail.com \\ ${ }_{2}^{2}$ Max-Planck-Institut für Radioastronomie, Bonn D-53123, Germany \\ ${ }^{3}$ National Astronomical Observatory of Japan, 2-21-1 Osawa, Mitaka, Tokyo 181-8588, Japan \\ ${ }^{4}$ Centre for Astronomy, Faculty of Physics, Astronomy, and Informatics, Nicolaus Copernicus University, Grudziadzka 5, 87-100 Torun, Poland \\ ${ }^{5}$ Osservatorio di Radio Astronomia (INAF-ORA), Via P. Gobetti 101, I-40129 Bologna, Italy \\ ${ }^{6}$ Harvard-Smithsonian Center for Astrophysics, Cambridge, Massachusetts 02138, USA \\ ${ }^{7}$ School of Physical Sciences, University of Kent, Ingram Building, Canterbury, Kent CT2 7NH, UK \\ ${ }^{8}$ School of Astronomy and Space Science, Nanjing University, Nanjing 210008, China \\ Received 2016 June 27; revised 2016 August 25; accepted 2016 August 27; published 2016 December 2
}

\begin{abstract}
We conducted Very Large Array C-configuration observations to measure positions and luminosities of Galactic Class II $6.7 \mathrm{GHz}$ methanol masers and their associated ultra-compact $\mathrm{H}$ II regions. The spectral resolution was $3.90625 \mathrm{kHz}$ and the continuum sensitivity reached $45 \mu \mathrm{Jy}_{\text {beam }}{ }^{-1}$. We mapped 372 methanol masers with peak flux densities of more than $2 \mathrm{Jy}$ selected from the literature. Absolute positions have nominal uncertainties of 0 "! 3 . In this first paper on the data analysis, we present three catalogs; the first gives information on the strongest feature of 367 methanol maser sources, and the second provides information on all detected maser spots. The third catalog presents derived data of the 127 radio continuum counterparts associated with maser sources. Our detection rate of radio continuum counterparts toward methanol masers is approximately one-third. Our catalogs list properties including distance, flux density, luminosity, and the distribution in the Galactic plane. We found no significant relationship between luminosities of masers and their associated radio continuum counterparts, however, the detection rate of radio continuum emission toward maser sources increases statistically with the maser luminosities.
\end{abstract}

Key words: catalogs - ISM: molecules - masers - stars: formation

Supporting material: figure sets, machine-readable tables

\section{INTRODUCTION}

Although massive stars make up only a few percent of the stellar population in the Milky Way, they play a central role in many astrophysical processes, such as shaping the interstellar medium, regulating star formation, and ultimately governing the evolution of their host galaxies (Kennicutt 2005). However, to date, understanding massive star formation remains a challenge because of numerous observational problems. Nevertheless, investigations of the natal environment of massive stars provide rich clues for a better understanding of its physical and chemical properties and the star formation process itself.

Massive star-forming regions (MSFRs) offer a variety of tracers that can be detected in different wavebands, including massive dense cores (Zinnecker \& Yorke 2007), infrared dark clouds (Kauffmann \& Pillai 2010), mid-infrared sources (Purcell et al. 2013), extended green objects (Cyganowski et al. 2008), molecular outflows (Beuther et al. 2002), masers (the most common species are hydroxyl, methanol, and water; Farmer 2014), and compact and ultra-compact (UC) H II regions (Churchwell 2002). Understanding what these signposts tell us may help us to construct an evolutionary sequence for young massive stars.

Methanol masers at $6.7 \mathrm{GHz}$ were discovered by Menten (1991); they, along with $22.2 \mathrm{GHz} \mathrm{H}_{2} \mathrm{O}$ masers, are the brightest and most widespread maser species in MSFRs (e.g., Caswell et al. 1995; Minier et al. 2003; Xu et al. 2008). These masers may be one of the earliest and ubiquitous tracers of the massive star formation process (Urquhart et al. 2015). Most $6.7 \mathrm{GHz}$ methanol masers have been observed with a variety of telescopes and with differing resolutions. For example, Pandian et al. (2011) observed 57 methanol masers at $6.7 \mathrm{GHz}$ with the
Multi-Element Radio-Linked Interferometer Network with an angular resolution of 60 mas. At this resolution they found a close correspondence between the masers and mid-infrared sources and claimed that their results supported the theoretical model that these masers are pumped by infrared dust emission in the vicinity of massive proto-stars.

Compact and UC H II regions are clear indicators of sites of massive stars (Hoare 2005), whose ionizing photons are provided by embedded OB stars in the later stages of massive star formation (Churchwell 2002). The thermal bremsstrahlung radiation from the ionized gas makes $\mathrm{H}$ II regions directly observable in radio continuum bands. In recent decades, a number of radio survey have identified many hundreds of UC H II regions. For example, Urquhart et al. (2009) observed 659 MSFR candidates with the Karl G. Jansky Very Large Array (VLA) at $4.9 \mathrm{GHz}$ and an angular resolution of 1 ".5, achieving rms noise levels of $\approx 0.2 \mathrm{mJy}$ beam $^{-1}$, and detected 391 compact or UC H II regions. Many observations (e.g., Caswell 2009; Caswell \& Breen 2010; Sanna et al. 2010) have established an association of these $\mathrm{H}$ II regions with $6.7 \mathrm{GHz}$ methanol masers. Since compact and UC H II regions have presented a reliable snapshot of MSFRs within the last $\sim 10^{5}$ years (Davies et al. 2011; Mottram et al. 2011), a census on this later phase of MSFRs can help us to better understand the evolutionary sequence of massive star formation and its association with different tracers.

In order to study the relationship of $6.7 \mathrm{GHz}$ methanol masers to compact and UC H II regions, we conducted a large survey to simultaneously observe the methanol maser line and C-band continuum emission with the VLA in its C-configuration. We mapped 372 class II methanol maser sources selected from literature, at $6.7 \mathrm{GHz}$ with peak fluxes 
$>2$ Jy. Absolute positions were established based on phasereferenced observations to astrometric calibrators. Our observations also served as precursor observations for the Bar and Spiral Structure Legacy (BeSSeL) Survey (Brunthaler et al. 2011) to provide high accuracy positions of maser sources necessary for VLBA observations.

Our uniform and high angular resolution survey includes the vast majority of known $6.7 \mathrm{GHz}$ methanol masers and their nearby compact or UC H II regions. The survey produced three catalogs: (1) a methanol maser catalog containing accurate positions, radial velocities, and flux densities of the brightest spots; (2) a catalog of maser spectra and spot maps; and (3) a catalog of $\mathrm{H}$ II regions toward the methanol masers.

Compact $\mathrm{H}$ II (referring to both compact and UC H II) regions and methanol masers offer a unique opportunity to peer into the deep interior of MSFRs at high angular resolution since at centimeter wavelengths their natal clouds are optically thin; the large database also should allow statistically meaningful estimates of many characteristics of $6.7 \mathrm{GHz}$ methanol masers and identified H II region counterparts. In combination with other signposts of massive star formations, the catalogs may provide important clues to solve several key issues related to massive star formation, such as whether $6.7 \mathrm{GHz}$ methanol masers are located in accretion disks or in outflow walls (or both), when in the process of star formation these two signposts occur, and how MSFRs evolve with time. Some prominent class II methanol masers are found in the dense molecular material associated with UC $\mathrm{HII}$ regions, $\mathrm{W} 3 \mathrm{OH}$ and NGC 7538 being prominent examples (Menten et al. 1988). However, most have no radio continuum emission at the few mJy level that could be easily detected with the original VLA or the ATCA (e.g., Walsh et al. 1998; Minier et al. 2001). This leaves open the question of whether weaker radio emission could be associated with masers at the level that could be expected from younger, hyper-compact H II regions.

In Section 2 we describe the sample and the design of the observations. The data analysis is described in Section 3. The three catalogs mentioned above are presented in Section 4. In Section 5, statistical properties of the $6.7 \mathrm{GHz}$ methanol masers and their associated compact $\mathrm{H}$ II regions are discussed. A brief summary is given in Section 6. This paper is the first in a series investigating massive star formation and its evolutionary sequence.

\section{OBSERVATION DESIGN}

\subsection{Sample}

We selected our sample of $6.7 \mathrm{GHz}$ methanol masers based on the following criteria:

1. Sources should have a decl. higher than $-30^{\circ}$ to ensure the visibility from the VLA site.

2. Since subsequent VLBA observations for the BeSSeL Survey require high signal-to-noise ratio $(\mathrm{S} / \mathrm{N})$ for parallax measurements, we required a peak flux density greater than $2 \mathrm{Jy}$.

Taking these constraints into account, 372 unique targets were selected from the following methanol maser surveys: the Methanol Multi-Beam catalog (MMB; Caswell \& Breen 2010; Green et al. 2010, 2012), the Arecibo Methanol Maser Galactic Plane Survey (AMGPS; Pandian et al. 2011), the Torun catalog of $6.7 \mathrm{GHz}$ methanol masers (Szymczak et al. 2012), and other individual observations of known $6.7 \mathrm{GHz}$ methanol masers or MSFRs (Caswell et al. 1995; Walsh et al. 1997, 1998; Xu et al. 2008; Caswell 2009). Green et al. (2009) stated that the MMB survey was complete to $\sim 80 \%$ at $0.8 \mathrm{Jy}$ and $\approx 100 \%$ at $1 \mathrm{Jy}$. At the time we started our observation, MMB had published sources in the Galactic longitude range from $186^{\circ}$ to $20^{\circ}$ and Galactic latitude range from $-2^{\circ}$ to $2^{\circ}$. However, most of the MMB Survey covers the Southern Galactic plane and is not visible to the VLA. Our observations included MMB sources in the Galactic longitude range from $357^{\circ}$ to $20^{\circ}$. With a flux cutoff at $2 \mathrm{Jy}$, we believe the sample in this area is complete. The AMGPS methanol maser survey uniformly sampled from $35^{\circ} .2 \leqslant l \leqslant 53^{\circ} .7$ with $|b| \leqslant 0.41$ and was complete to $0.27 \mathrm{Jy}$ (Pandian et al. 2007). Thus, our catalog is also complete to $2 \mathrm{Jy}$ in the AMGPS area. The completeness of the Torun catalog varies in Galactic longitude, however, Szymczak et al. (2012) concluded that all maser sources brighter than $7.5 \mathrm{Jy}$ in the peak were included. Thus, our survey is not complete to $2 \mathrm{Jy}$ for these sources, nor from sources that were from targeted observations toward individual objects. Most of the masers are located in the first and second quadrants of the Galactic plane.

\subsection{Observational Design}

The observations were conducted with the VLA in C-configuration using five sessions from 2012 February 28 to April 16. At the beginning of each observation, a primary flux calibrator $^{9}$ was observed to establish amplitude calibration, followed by observations alternating between phase calibrator and target sources.

We adopted complex gain calibrators from the catalogs of Immer et al. (2011) and the first realization of the International Celestial Reference Frame (ICRF1, Ma 1997). Phase calibrators were chosen to be within $5^{\circ}$ of the targets and were observed at the beginning and end of each block. A typical cycle consisted of a scan of the phase calibrator, followed by a group of snapshots of nearby targets, then a revisit of the phase calibrator.

Spectral line data used 2048 channels across $8 \mathrm{MHz}$, yielding a channel spacing of $3.90625 \mathrm{kHz}$ at the central frequency of $6.6685192 \mathrm{GHz}$ and a velocity resolution of $0.176 \mathrm{~km} \mathrm{~s}^{-1}$. The continuum observations employed two $1 \mathrm{GHz}$ sub-bands from 4.9840 to $6.0080 \mathrm{GHz}$ (hereafter, the low band) and from 6.6245 to $7.6485 \mathrm{GHz}$ (hereafter, the high band) and each sub-band was divided into 16 channels. Snapshot observations of the maser sources achieved about $20 \mathrm{~s}$ on source and reached typical noise levels of $45 \mu \mathrm{Jy}_{\text {beam }}{ }^{-1}$ for the continuum maps and $33 \mathrm{mJy}$ beam $^{-1}$ per channel for the spectral line maps. Technical details of the observations are given in Table 1.

\section{DATA REDUCTION}

\subsection{Methanol Maser Line}

Maser data were calibrated through a standard spectral line data reduction procedure in the Astronomical Image Processing System (AIPS). In brief, we first flagged suspiciously identified entries by eye, then determined the flux density scale based on

\footnotetext{
9 Calibrators used were $3 \mathrm{C} 147,3 \mathrm{C} 286$, and 3C48. Their assumed flux densities were determined by the radio flux calibrator model files delivered with the Common Astronomy Software Applications (CASA).
} 
Table 1

Summary of Technical Details of the Observation

\begin{tabular}{ll}
\hline \hline Dates of observations & 2012 Feb. 28 \\
& 2012 Mar. 04 \\
& 2012 Mar. 07 \\
& 2012 Mar. 18 \\
& 2012 Apr. 16 \\
& C-configuration \\
Configuration & 27 \\
Antennae & 8 -bits \\
Digital sampler & $20 \mathrm{~s}$ \\
Average on-source time & $3^{\prime \prime} \times 6^{\prime \prime}$ \\
Synthesized beam & $\sim 10^{\prime} \times 10^{\prime}$ \\
Field of view & Line: $6664.5-6672.5 \mathrm{MHz}$ \\
Frequency coverage & Cont.: $4984.0-6008.0 \mathrm{MHz}$ \\
& and $6.6245-7.6485 \mathrm{MHz}$ \\
\hline
\end{tabular}

the primary flux calibrators. Complex gains of secondary calibrators were then calculated and interpolated to the maser sources. We extracted a scalar averaged spectrum from the visibility data of each target in order to find the peak emission channel. Then we made an image from that channel's data covering $5 ! 12 \times 5 ! 12$ on the sky, and a detection was confirmed by requiring the peak brightness to exceed five times the rms noise level of the image. We then generated a spectroscopic image cube with a typical CLEAN restoring beam size of $3^{\prime \prime} \times 6^{\prime \prime}$. The positions of the maser spots were derived from these cubes.

In a small number of cases (33), we failed to detect maser emission in spite of the fact that maser sources were reported there in the literature. Positions for the centers of these fields are given in Table 2. In all cases we made maps of $5 \times 5$ arcmin. We suspect that many of these non-detections result from previous catalogs having identified telescope side lobes from a strong source at a position far removed from the true source. However, for some of the weaker sources, it is possible that time variability could explain our lack of detection $(<5 \sigma$ i.e., about $170 \mathrm{mJy})$. Repeated observations with longer integration might reveal whether variability explains the nondetections.

We improved the calibration of the maser sources using the brightest maser spot to self-calibrate phase and amplitude and then applied these corrections to all channels. For a maser spot with a peak flux density $>3 \mathrm{Jy}$, self-calibration improved the dynamic range of the maser image cube. To extract maser spots from image cubes, we ran the SAD task on each channel with a dynamic range cutoff at $10 \%$ of the image peak. In some cases, more than one maser source appeared in a field. When the separations exceeded $30^{\prime \prime}$, we labeled them as separate maser sources. The spectrum for each maser source was generated from the SAD output results and has a velocity resolution of $0.176 \mathrm{~km} \mathrm{~s}^{-1}$.

\subsection{Continuum}

We processed the continuum data using the Common Astronomy Software Applications (CASA). Our calibration procedure can be summarized as follows. After inspecting and flagging the data set, the flux density scale was set to the primary calibrator, bandpass- and antenna-based delays were then calculated for the primary calibrator and applied to the secondary calibrators and targets, we then finished the calibration by interpolating the amplitude and phase correction
Table 2

Non-detections

\begin{tabular}{|c|c|c|c|}
\hline Source Name & $\begin{array}{l}\text { R.A. } \\
\text { (hh:mm:ss.sss) }\end{array}$ & $\begin{array}{l}\text { Decl. } \\
\text { (ddd:mm:ss.sss) }\end{array}$ & Reference \\
\hline G000.69-0.04 & $17: 47: 24.74$ & $-28: 21: 43.6$ & $\mathrm{C} 10$ \\
\hline G000.71-0.03 & $17: 47: 25$ & $-28: 21: 00$ & W97 \\
\hline $\mathrm{G} 002.62+0.14$ & $17: 51: 12$ & $-26: 37: 00$ & W97 \\
\hline G010.00-0.03 & 18:07:52 & $-20: 18: 00$ & W97 \\
\hline G010.20-0.35 & $18: 09: 28.43$ & $-20: 16: 42.5$ & G10 \\
\hline G012.86-0.27 & $18: 14: 36.42$ & $-17: 54: 50.2$ & S14 \\
\hline G016.60-0.04 & $18: 21: 09$ & $-14: 31: 00$ & W97 \\
\hline G016.88-2.15 & $18: 29: 25$ & $-15: 15: 00$ & W97 \\
\hline G018.67+0.03 & $18: 24: 53.78$ & $-12: 39: 20.40$ & G10 \\
\hline G019.37-0.02 & $18: 26: 24$ & $-12: 03: 00$ & W97 \\
\hline G023.09-0.39 & $18: 34: 45.71$ & $-08: 55: 55.2$ & S14 \\
\hline G023.45-0.18 & $18: 34: 39$ & $-08: 31: 00$ & W97 \\
\hline G023.46-0.16 & $18: 34: 35.95$ & $-08: 29: 32.1$ & S14 \\
\hline G023.71-0.19 & $18: 35: 11$ & $-08: 17: 00$ & W97 \\
\hline $\mathrm{G} 025.23+0.29$ & 18:36:17.10 & $-06: 43: 11.8$ & S14 \\
\hline G025.66+1.06 & $18: 34: 20$ & $-05: 59: 00$ & W97 \\
\hline $\mathrm{G} 025.70+0.03$ & 18:38:05 & $-06: 25: 00$ & W97 \\
\hline G025.79-0.14 & $18: 38: 52$ & $-06: 25: 00$ & W97 \\
\hline G025.80-0.16 & 18:38:56.40 & $-06: 24: 52.7$ & SZ12 \\
\hline G025.81-0.04 & $18: 38: 32.02$ & $-06: 21: 28.6$ & S14 \\
\hline $\mathrm{G} 027.56+0.08$ & $18: 41: 20.26$ & $-04: 44: 25.0$ & S14 \\
\hline G028.31-0.37 & $18: 44: 19$ & $-04: 17: 00$ & W97 \\
\hline G029.91-0.05 & $18: 46: 05.9$ & $-02: 42: 27$ & X08 \\
\hline $\mathrm{G} 030.52+0.10$ & $18: 46: 41.37$ & $-02: 06: 07.6$ & SZ12 \\
\hline $\mathrm{G} 030.79+0.20$ & $18: 46: 48$ & $-01: 48: 46$ & W98 \\
\hline $\mathrm{G} 030.82+0.27$ & $18: 46: 37$ & $-01: 45: 00$ & W97 \\
\hline $\mathrm{G} 031.28+0.07$ & 18:48:10.02 & $-01: 26: 31.6$ & SZ12 \\
\hline $\mathrm{G} 031.42+0.32$ & $18: 47: 33$ & $-01: 12: 00$ & W97 \\
\hline G032.77-0.06 & $18: 51: 21.60$ & $-00: 10: 04.9$ & S14 \\
\hline G049.27-0.34 & 19:23:06.95 & $+14: 20: 10.9$ & S14 \\
\hline G049.67-0.46 & 19:24:19.60 & $+14: 38: 03.9$ & SZ12 \\
\hline $\mathrm{G} 189.77+0.34$ & 06:08:32.40 & $+20: 39: 25.3$ & SZ12 \\
\hline G358.39-0.48 & $17: 43: 37.83$ & $-30: 33: 51.1$ & $\mathrm{C} 10$ \\
\hline
\end{tabular}

Note. Source names and coordinates are taken directly from the references. The J2000 coordinates are presented in sexagesimal in corresponding units. Accuracy of the coordinates varies in different references. Fluxes are the peak values that are taken directly from the references. Reference abbreviations are: C10, Caswell \& Breen (2010); G10, Green et al. (2010); S14, Sun et al. (2014); SZ12, Szymczak et al. (2012); W97, Walsh et al. (1997); and X09, Xu et al. (2009).

(This table is available in machine-readable form.)

from secondary calibrators to targets. After some additional flagging, images were produced with a pixel size of 0 "! 3 . Most continuum images are $1024 \times 1024$ pixels; for some cases larger images were produced to include nearby strong sources to suppress the interference from them. Since there is considerable radio frequency interference at $\mathrm{C}$-band at the VLA site, we had to manually flag a considerable amount of data. In some cases this significantly increased the noise level in the final image. In addition, the flagging, together with snapshot observing, produced limited (u, v) coverage, which led to large side lobes in the final images, especially for sources at low decl.

The final maps were made with the CLEAN task in CASA, using the "multi-frequency synthesis" algorithm. Three maps were produced for each source, including those derived separately for the low- and high-band data and a combined map. We excluded channels near the $6.7 \mathrm{GHz}$ maser line when making these maps. Typical noise levels for the combined map 

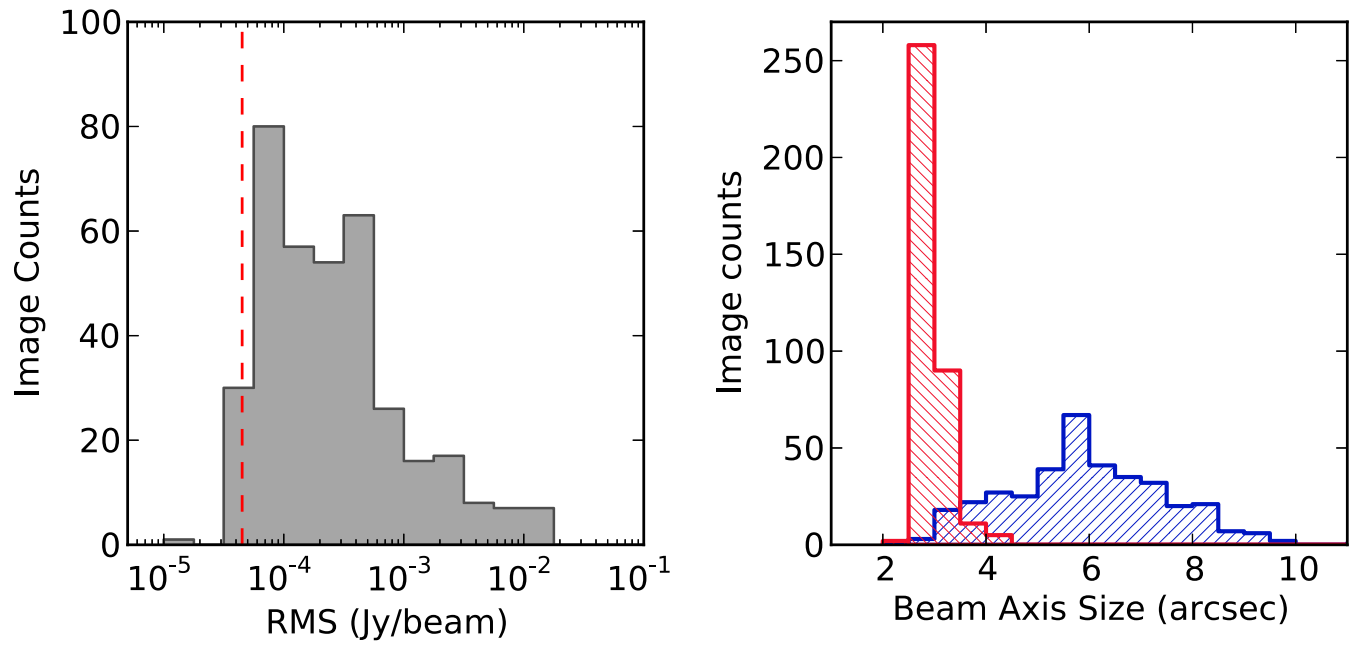

Figure 1. Statistical properties of the continuum images. Left panel: the gray histogram indicates the distribution of rms map noise, and the vertical red dashed line shows the expected thermal noise limit. Right panel: the distribution of the major (blue) and minor (red) axis FWHM sizes of the Gaussian restoring beams. A typical beam size for the survey is $6^{\prime \prime} \times 3^{\prime \prime}$.

Table 3

6.7 GHz Methanol Maser Catalog

\begin{tabular}{|c|c|c|c|c|c|c|c|c|c|}
\hline Source name & $\begin{array}{c}\text { R.A. } \\
\text { (hh:mm:ss.sss) }\end{array}$ & $\begin{array}{c}\text { Decl. } \\
\text { (dd:mm:ss.ss) }\end{array}$ & $\begin{array}{c}V_{\mathrm{lsr}} \\
\left(\mathrm{km} \mathrm{s}^{-1}\right)\end{array}$ & $\begin{array}{c}\Delta V \\
\left(\mathrm{~km} \mathrm{~s}^{-1}\right)\end{array}$ & $\begin{array}{c}S_{\text {peak }} \\
\left(\text { Jy beam }^{-1}\right)\end{array}$ & $\begin{array}{l}S_{\text {int }} \\
(\mathrm{Jy})\end{array}$ & $\begin{array}{l}\text { Distance } \\
(\mathrm{kpc})\end{array}$ & Prob. & Code \\
\hline G000.546-0.851 & $17: 50: 14.523$ & $-28: 54: 31.25$ & 15.06 & 8.95 & 90.75 & 1091.44 & $2.7 \pm 0.3$ & 0.59 & $\mathrm{~A}$ \\
\hline G000.644-0.042 & $17: 47: 18.663$ & $-28: 24: 24.82$ & 49.95 & 6.67 & 55.06 & 497.05 & $10.8 \pm 0.2$ & 0.54 & B \\
\hline G000.651-0.048 & $17: 47: 21.125$ & $-28: 24: 18.12$ & 47.92 & 8.96 & 15.45 & 73.89 & $8.3 \pm 0.3$ & 0.54 & $\mathrm{~N}$ \\
\hline G000.656-0.040 & $17: 47: 20.062$ & $-28: 23: 46.65$ & 52.42 & 5.62 & 6.47 & 35.99 & $10.8 \pm 0.2$ & 0.63 & A \\
\hline
\end{tabular}

Note. This is a sample of the $6.7 \mathrm{GHz}$ methanol maser catalog. Source names are based on the Galactic coordinates. J2000 coordinates are presented in sexagesimal terms. $V_{1 \mathrm{sr}}$ is the radial velocity of the strongest spot of the source. $S_{\text {peak }}$ and $S_{\text {int }}$ are the peak and integrated fluxes. Distances with uncertainties are presented in kpc. The probabilities evaluate how likely the sources are located on the given distances. See Section 5.2 for details. In the last column, code indicates the association between maser source and the continuum emission. "A" means the associated continuum emission is detected and classified as group A, "B" means continuum emission is found in the vicinity of the maser but with no evidence supporting a physical relationship between the maser and the continuum source. " $\mathrm{N}$ " represents the absence of continuum emission.

(This table is available in its entirety in machine-readable form.)

were $\sim 50 \mu \mathrm{Jy}_{\text {beam }}{ }^{-1}$. The left panel of Figure 1 shows the distribution of rms noise levels for the combined maps. The peak of the histogram is comparable to the theoretical noise level $\left(\sim 45 \mu \mathrm{Jy}_{\text {beam }}{ }^{-1}\right)$ for our observations as calculated by the VLA Exposure Calculator. ${ }^{10}$ For maps that have much greater noise than expected, most were affected by side lobes of nearby strong sources and complications from strong diffuse background emission.

In order to extract positions of radio continuum sources in the fields, we used the SExtractor algorithm (Bertin \& Arnouts 1996) to automatically find and measure compact radio sources. Although, SExtractor was developed for source extraction in visible or infrared images, it has been successfully used for radio sources in the past (Thompson et al. 2006; Contreras et al. 2013; Urquhart et al. 2015). The continuum sources identified by SExtractor were filtered before inclusion in our catalog. We required the peak flux to be above $3 \sigma$ of the rms noise of the integrated map, or above $2 \sigma$ on each of the three images (i.e., the integrated image, the high-band image, and the low-band image). Under these constraints, 349 continuum sources were identified. For the interest of investigation of maser to continuum source associations, we

\footnotetext{
${ }^{10}$ https://obs.vla.nrao.edu/ect/
}

adopted only continuum source with the smallest angular offset to the strongest maser spot in each maser source, if multiple continuum sources appear in the same field. In all, 279 sources were adopted, making up the continuum source catalog.

\section{SURVEY PRODUCTS}

\subsection{VLA $6.7 \mathrm{GHz}$ Methanol Maser Catalog}

Our VLA $6.7 \mathrm{GHz}$ methanol maser catalog in Table 3 includes 367 methanol maser sources, sorted by Galactic longitude, with $\approx 0$ "' 3 accurate J2000 coordinates, peak $\left(S_{\text {peak }}\right)$ and integrated $\left(S_{\text {int }}\right)$ flux densities, local standard of rest (LSR) velocities $\left(V_{1 \mathrm{sr}}\right)$ for the strongest spots, the velocity ranges of masers $(\Delta V)$, and the estimated heliocentric distance and its probability (see Section 5.2 for details). The LSR velocity and peak and integrated flux densities were estimated by fitting two-dimensional Gaussian brightness distributions of the reference spot.

Positions of the brightest, compact maser spots were determined by phase calibrator observations prior to any selfcalibration. The formal precision in determining position is directly proportional to the synthesized beam size and inversely to the S/N (see Reid et al. 1988, Equation (1)). For the typical beam size $3^{\prime \prime}(\mathrm{E}-\mathrm{W}), 6^{\prime \prime}(\mathrm{N}-\mathrm{S})$ and $\mathrm{S} / \mathrm{N}$ value of 50 , in our 
Table 4

Maser Spot Catalog

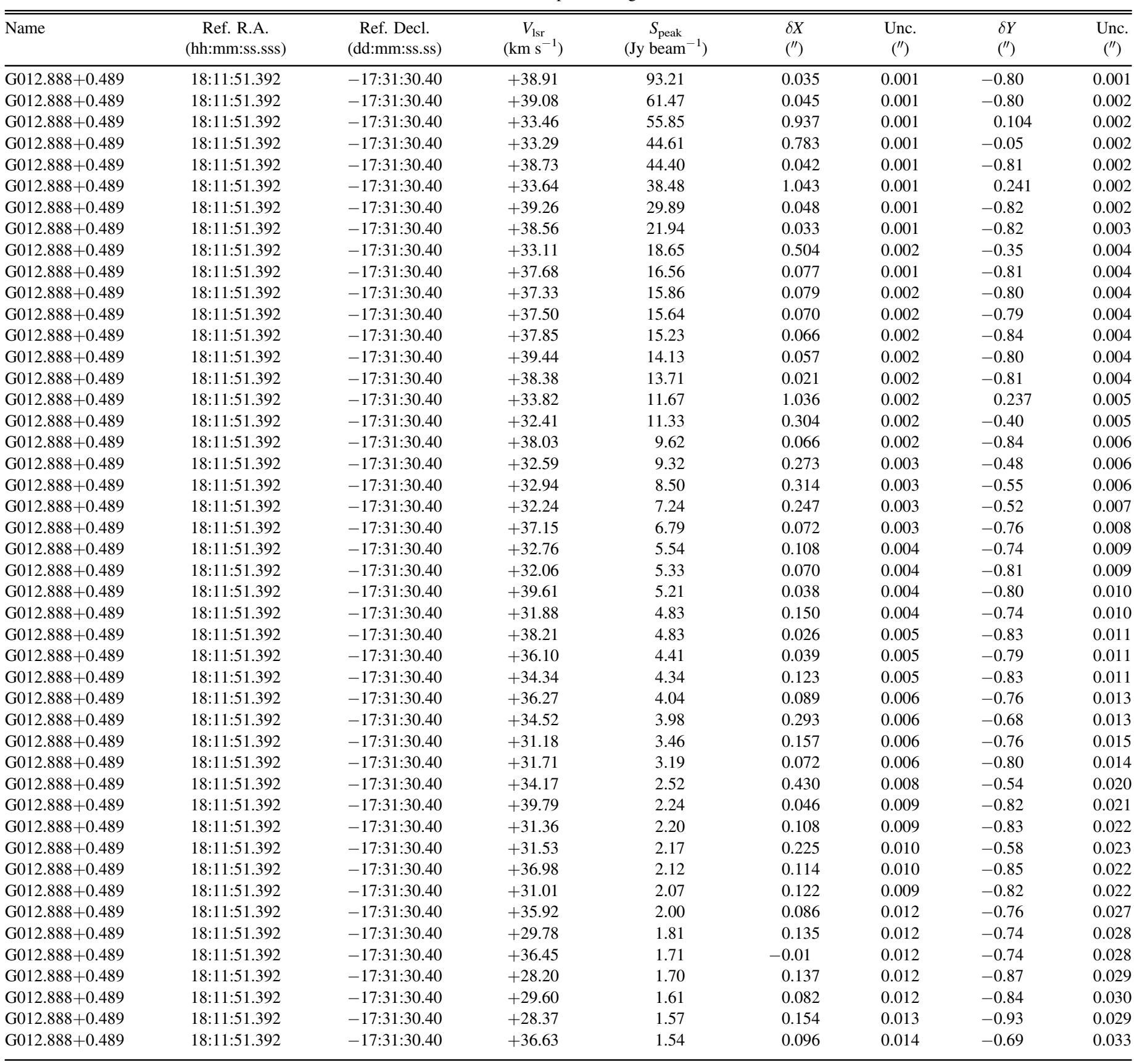

Note. This table demonstrates the maser spots in source G012.888+0.489 as an example of the spot catalogs. The name of the source and the reference point are given in the first three columns. In the body of the table, each entry is representing one spot, given in order from brightest to faintest. Radial velocities and peak fluxes from Gaussian fitting resulta are given in the second and third columns. Columns 6-9 are the offsets of the spot relative to the reference point in arcseconds. The full-size methanol maser spots catalog for all sources will be available in machine-readable format via online material.

(This table is available in its entirety in machine-readable form.)

observation, the position precision is $\approx 27$ mas in the $\mathrm{E}-\mathrm{W}$ direction and $\approx 54$ mas in the $\mathrm{N}-\mathrm{S}$ direction. Thus we list the $\mathrm{R}$. A. of maser spots to three decimal places in seconds, and decl. to two decimal places in arcseconds in the catalog. We have taken these values as the formal precision of maser spots' positions. However, uncompensated atmospheric delays likely make the random error much smaller than the systematic error. Based on experience with snapshot imaging of $\mathrm{OH}$ masers by Argon et al. (2000) and a comparison with maser positions determined from VLBI observations (see Section 5.1 for details), we adopt a realistic total position accuracy of \pm 0 "' 3 .

\subsection{Methanol Maser Spot Catalog}

Maser spots extracted from line cubes constitute a second catalog. In order to facilitate the investigation in morphology and kinematics of individual maser sources, we present maser spots arranged by the hosting maser source name in Table 4. In 
Table 5

Catalog of Radio Continuum Counterparts of Maser Sources

\begin{tabular}{|c|c|c|c|c|c|c|c|c|c|c|}
\hline Source Name & $\begin{array}{c}\text { R.A. } \\
\text { (hh:mm:ss.ss) }\end{array}$ & $\begin{array}{c}\text { Decl. } \\
\text { (dd:mm:ss.s) }\end{array}$ & $\begin{array}{c}F_{\text {int }} \\
(\mathrm{mJy})\end{array}$ & $I / P$ & $\begin{array}{c}a \\
\left({ }^{\prime \prime}\right)\end{array}$ & $\begin{array}{l}b \\
(")\end{array}$ & $\begin{array}{l}\text { P.A. } \\
\left({ }^{\circ}\right)\end{array}$ & $\begin{array}{l}\text { Offset } \\
(\mathrm{pc})\end{array}$ & $\begin{array}{l}\text { Diameter } \\
(\mathrm{pc})\end{array}$ & Group \\
\hline G001.328+0.149 & 17:48:09.95 & $-27: 43: 09.0$ & 138.00 & 59.87 & 44.8 & 41.7 & 1.9 & 0.27 & 0.91 & $\mathrm{~A}$ \\
\hline G002.143+0.009 & $17: 50: 36.00$ & $-27: 05: 47.4$ & 5.87 & 1.12 & 2.4 & 1.2 & 14.0 & 0.06 & 0.12 & A \\
\hline G003.910+0.001 & 17:54:38.77 & $-25: 34: 45.1$ & 49.70 & 1.03 & 8.4 & 2.9 & 174.4 & 0.02 & $<0.45$ & A \\
\hline G004.393+0.078 & $17: 55: 21.59$ & $-25: 06: 12.5$ & 124.00 & 28.42 & 52.3 & 26.9 & 139.4 & 6.38 & 3.71 & B \\
\hline
\end{tabular}

Note. The names of the corresponding maser sources are listed in the first column. Given, from the second to fourth columns, are J2000 coordinates, the integrated flux of the entire continuum source $F_{\text {int }}$ and $I / P$, the ratio of integrated flux to peak pixel value. The shape of the source is represented by elliptic parameters $a, b$, and position angle. Offset indicates the displacement between peaks of maser and continuum sources in pc. The diameter column indicates the diameter of the continuum source derived from the major axis $a$ and the heliocentric distance of the associated maser. Note that the values in this column with " $<$ " represent the upper limit of the physical diameter of the corresponding continuum source due to our limited resolving power. The last column implies the association type of the continuum source.

(This table is available in its entirety in machine-readable form.)
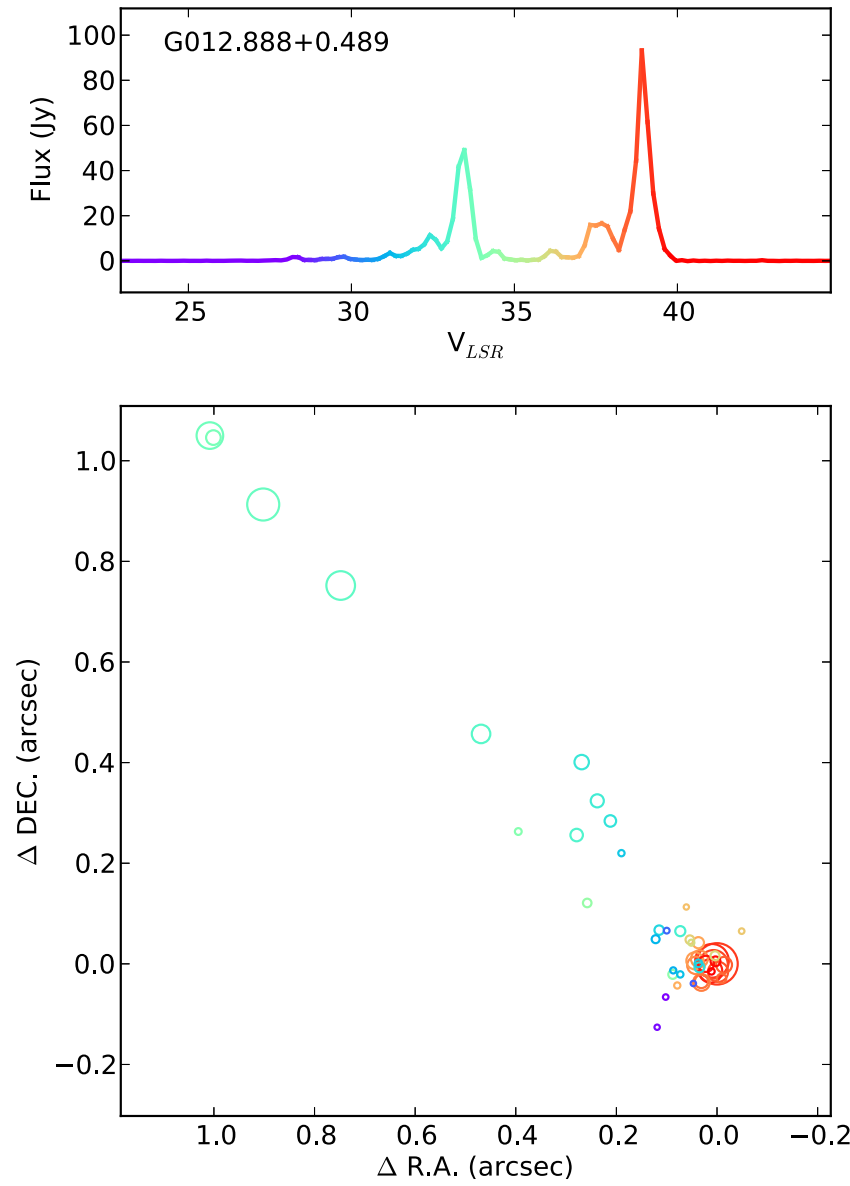

Figure 2. An example of the $6.7 \mathrm{GHz}$ maser data for G012.888+0.489. The upper panel shows the maser source spectrum and the lower panel shows a map of the maser spots. Both plots have color-coded radial velocities. The diameter of each spot is proportional to the flux. Positional offsets are relative to the strongest maser spot. Spectra and maps for all sources are available as online material.

(The complete figure set (367 images) is available.)

this spot catalog, columns in the catalog are maser source name, reference position of each source, LSR velocity, peak flux density, and the offsets of spot from the reference position with their uncertainty. The reference positions are determined

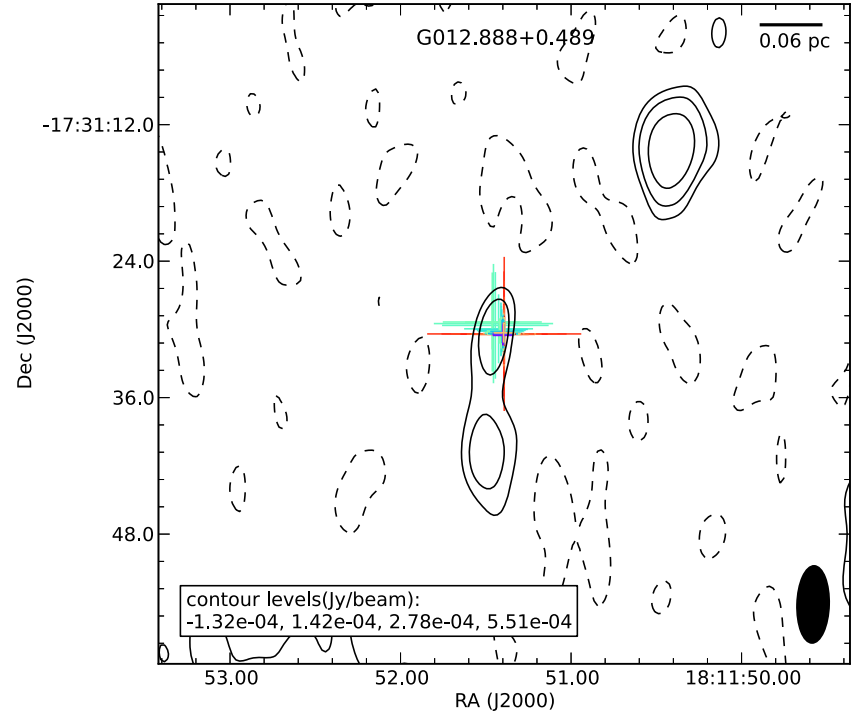

Figure 3. An example radio continuum map (contours) with maser spots (crosses) overlaid for G012.888+0.489. Colors of the crosses indicate LSR velocity, as indicated in the source spectrum in Figure 2. For strong sources with peak brightness greater than $8 \sigma$, the contour levels are logarithmic at $2 \sigma$, $4 \sigma, 8 \sigma, 16 \sigma$, etc. For fainter sources the contour levels are $2 \sigma, 4 \sigma, 6 \sigma, 8 \sigma$, etc. Negative map brightnesses are indicated with dashed contours. The FWHM of the restoring beam is indicated by the black ellipse in the lower right corner. A linear length scale based on the estimated heliocentric distance of the maser is shown in the upper right corner.

(The complete figure set (367 images) is available.)

conventionally close to the strongest maser spot in each source, thus they bear no actual physical meaning. One should also note that, in the catalog, the offsets of individual maser spots are provided uniformly by the SAD task in AIPS, which are listed to three decimal places in arcseconds. However, as the $\mathrm{S} / \mathrm{N}$ of the single channel image varies from channel to channel and source to source, such a presentation may not indicate the actual precision of individual measurements. The nominal precision of maser sources is $\approx 27$ mas in the E-W direction and $\approx 54$ mas in the N-S direction (See Section 4.1). From this catalog, we produced a spot map image and a spectrum for each maser source. See Figure 2 for an example. The full-size machine-readable spot catalog and images for 367 sources are available as online material. 

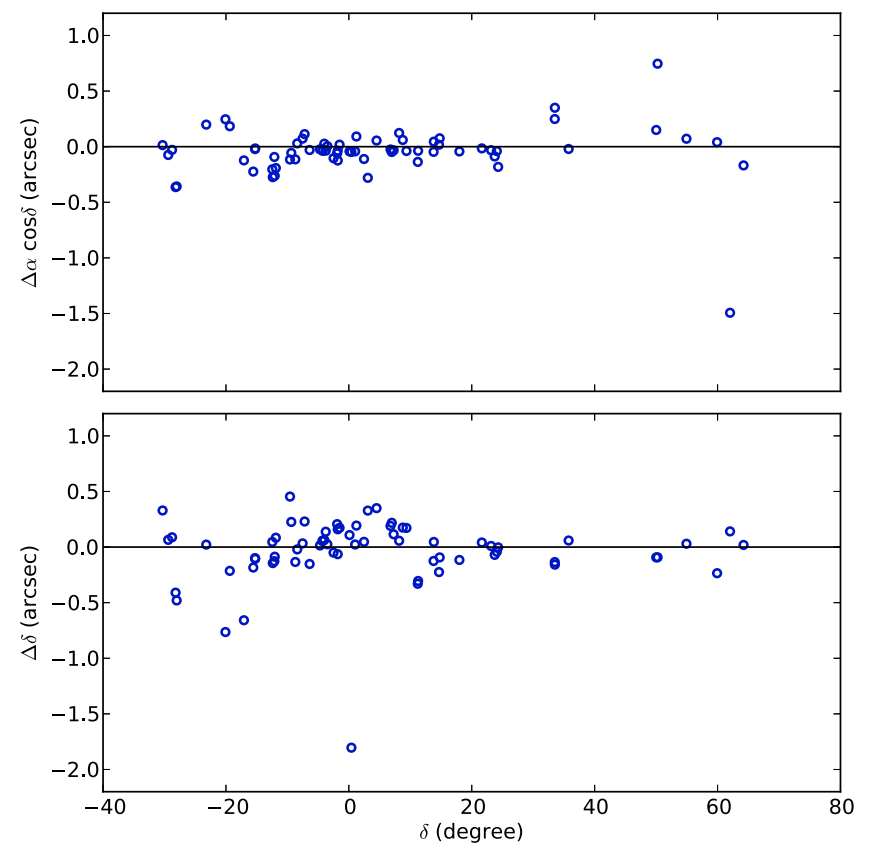

Figure 4. Position error as a function of source decl., obtained by subtracting high accuracy BeSSeL Survey VLBI positions from our VLA maser positions. Upper panel: E-W $(\Delta \alpha \cos \delta)$ errors with an rms 0 ". 23 ; they show no significant trend with source decl. (beyond two outliers). Lower panel: $\mathrm{N}-\mathrm{S}(\Delta \delta)$ errors with an rms of 0 !" 30 ; they show a possible increase below a decl. of $-10^{\circ}$.

\subsection{Continuum Catalog}

A catalog of radio continuum sources nearest the masers is presented in Table 5, including the Galactic source name, J2000 coordinates of the peak emission, integrated flux densities, the ratio of integrated flux to peak pixel value, ellipse parameters describing source's shape, the spatial offset between peak of the continuum source and the corresponding maser, the diameter of continuum source derived directly from the semimajor axis length, and a "Group" code. The precision of continuum peak position comes from the similar deduction we have made for the maser spots. As the typical $\mathrm{S} / \mathrm{N}$ value of continuum images is 10 , the positional precisions on $\mathrm{E}-\mathrm{W}$ and $\mathrm{N}-\mathrm{S}$ direction are $\approx 135$ mas and $\approx 270$ mas. The integrated flux density $\left(F_{\text {int }}\right)$ is derived from 2 D Gaussian fitting of the continuum brightness distribution profile. The ratio of integrated flux to peak pixel value provides a measure of the compactness of the source, which is useful for morphological analysis of the continuum sources. The ellipse parameters $a, b$, and $\theta$ are the length of the major and minor axes and the positional angle counting east of north, respectively. Elliptical parameters are derived from the 2D Gaussian fitting that is deconvolved from the restoring beam if the source is resolved on both the major and minor axis directions; otherwise, parameters are of the 2D Gaussian component convolved with the restoring beam. We regard them as estimates of a source's angular size, and the parameter $a$ is used to calculate a spatial diameter using the heliocentric distance of the associated maser. However, our observations are lacking sufficient resolving power to measure the actual size of some extremely compact and distant sources. We indicate unresolved sources with a " $<$ " symbol when the formal fitted FWHM is smaller than the CLEAN restoring beam in any direction.



Figure 5. Galactic plane distribution of methanol masers, with (color-coded) spiral arm associations and distances estimated by the Bayesian approach of Reid et al. (2016). Error bars indicate the formal uncertainty of the distance estimates. Gray circles indicate the small number of sources for which no significant arm assignment could be made.

We divide continuum sources into two groups: Group A includes 140 continuum sources that are associated with a methanol maser, whereas 139 Group B sources have no associated maser sources. See Section 5.3 for a more detailed discussion of this issue.

We overlaid the maser spots on the integrated continuum contour map to give a direct illustration of the positional relation of these two kinds of detections. These images can be found in the online material; one example is shown in Figure 3 in which the colored crosses are the maser spots and the black contours indicate the radio continuum emission.

\section{SOURCE PROPERTIES}

\subsection{Positions}

The absolute positions of masers come from the images without self-calibration. We estimate position uncertainty by comparing our result with the VLBI measurement from the first epoch result of BeSSeL Survey ${ }^{11}$ observed by VLBA and having better than 10 mas accuracy. Figure 4 shows the differences between the sources in common to the two surveys,

\footnotetext{
$\overline{{ }^{11} \text { http://bessel.vlbi-astrometry.org/first_epoch }}$
} 


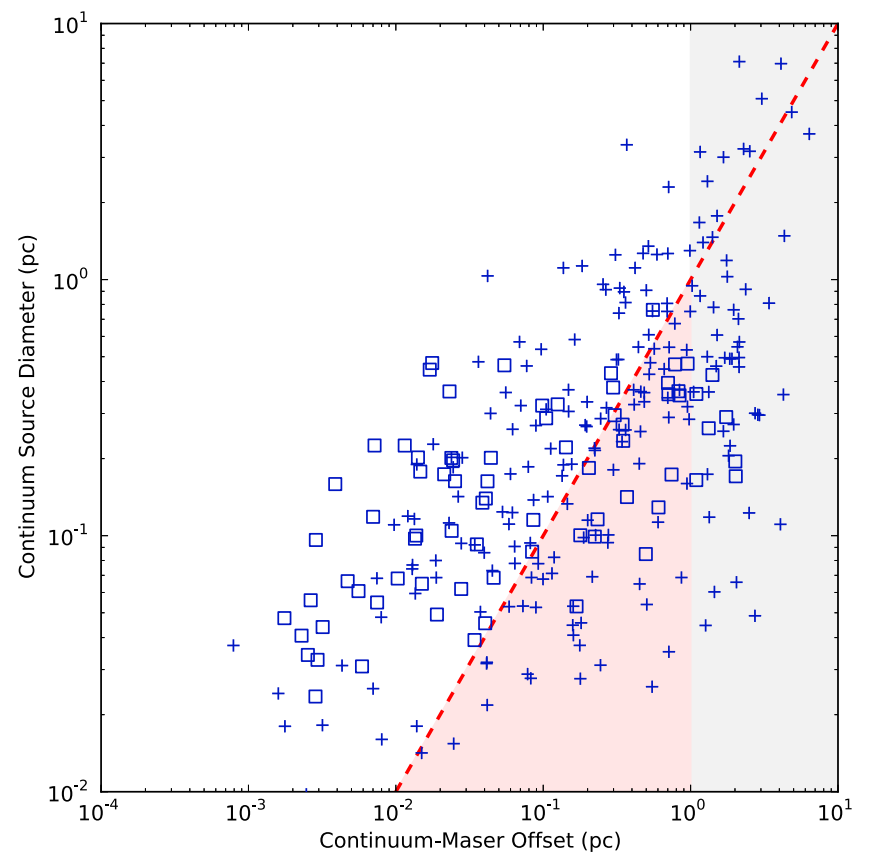

Figure 6. Continuum source diameter vs. the offset between peak positions of the continuum and reference maser spot emission. Blue crosses indicate continuum sources with size measurements, while blue squares indicate sources with only upper limits on size. The red dashed line traces where the continuum source size equals its offset from the maser. The gray shaded region indicates separations $>1 \mathrm{pc}$, which likely indicates no direct physical association. Sources in the white area comprise Group A (likely associated), and those in pink and gray area comprise Group B (not likely associated).

which we attribute to errors in the VLA data. In E-W direction, the rms errors are about $0 . ! 23$, and in the $\mathrm{N}-\mathrm{S}$ direction they are about 0 ". 30, and somewhat larger for $\delta<-10^{\circ}$. The positional uncertainty of masers is mainly limited by atmospheric irregularities that are only partially canceled by the small separation $\left(<5^{\circ}\right)$ between phase calibrators and targets.

\subsection{Distance}

Reid et al. (2014) have published a summary of the parallax measurements of masers that are hosted by Galactic MSFRs. Parallax measurements do exist for a total of 53 of our sources. While in principle kinematic distance can be calculated for sources in the Galactic plane, these can be very inaccurate in the general direction of the Galactic center and anti-center directions and within the solar circle they are multi-valued, i.e., are affected by the "kinematic distance ambiguity," KDA. In addition, kinematic distance estimates depend on the assumed Galactic rotation curve, as well as the assumption of circular Galactic orbits.

Reid et al. (2016) proposed an alternative approach that leverages VLBI parallax measurements of MSFRs from the BeSSeL Survey and the Japanese VERA project to significantly improve the accuracy and reliability of distance estimates to any source known to follow a spiral structure. Their Bayesian approach considers the likelihood of a spiral arm assignment, the angular displacement from the Galactic plane, the proximity to individual sources with parallax measurements, as well as kinematic information to estimate a full distance probability density function for each source. Since this method not only synthesizes different approaches of distance determination but also shows good agreement with



Figure 7. Continuum peak and integrated flux density plotted as a function of maser peak flux density. Sources in Group A and B are denoted by red and blue circles, respectively. No relation between continuum and maser strengths is apparent.

results from the $\mathrm{HI}$ absorption method when resolving the KDA and can be applied to the full Galactic longitude range, we adopt it to estimate distances (and uncertainties) to the 367 methanol maser sources in our catalog based on the radio velocity of the reference spot in each source. However, one should note that the determination of the distances strongly depends on the spiral arm assignment. We simply take the association with the highest probability, so the distances of some sources may be more uncertain than what is claimed by the nominal uncertainty. In the methanol maser catalog the probability of the most likely arm assignment is given. We suggest that one should take special care when dealing with those low probability distances and their subsequent parameters, such as luminosities, spatial sizes, etc.

In Figure 5, we show the distribution of all maser sources projected on the Galactic plane. Note, however, that the spiral arm models used to partially constrain distances are assumed to be correct; the accuracy of these models is limited to regions where parallaxes have been measured (see Reid et al. 2016 for the details).

\subsection{Association of Masers and Continuum Sources}

We establish an association between a maser and a continuum source based on their projected separations and 
the size of the continuum source. Since the two well-accepted pumping mechanisms of the class II methanol maser are infrared radiation from the central YSO (Sobolev \& Deguchi 1994) and collision (Cragg et al. 2005), the continuumassociated class II methanol masers can only exist in the natal molecular cloud of the HC/UC H II region. Projected on the sky, the associated maser should appear within or in the vicinity of the area of the continuum source. Thus, we introduce two criteria on the projected spatial distance between peak positions of the maser and continuum sources when considering their association. First, since the median value of the radii of gas clumps hosting methanol masers is $0.97 \mathrm{pc}$ (Urquhart et al. 2013), we assume the maser source is not physically associated with the continuum if their projected offset is greater than $1 \mathrm{pc}$. Second, two objects are considered associated with each other only when the offset is smaller than the semimajor axis of the continuum source. In Figure 6, we plot the continuum source diameter versus the offset to the maser reference spot. The gray area in the plot represents the offset larger than $1 \mathrm{pc}$, while the red dashed line indicates that the continuum source diameter equals the projected offset to the maser. The markers in gray area are denoting the maser-continuum pairs that are not in the same molecular cloud, and those in the pink area can be interpreted as the continuum source and the methanol maser tracing different massive star formation sites in the same molecular cloud. In other words, the maser-continuum pair in these two areas show no possibility of direct physical association. We refer to sources in these two area as Group B. In total, 139 sources are found in this group. In contrast, we assign 140 continuum sources in the white area of the plot (i.e., offsets less than either $1 \mathrm{pc}$ or the diameter of continuum source) as physically associated with their counterpart masers and refer to these as Group A. Here we provide a rather rough classification. A more comprehensive diagnosis of the association is still in need in follow-up work since the relationship of these two signposts will be of great importance to the study of the evolutionary stages of HMSFRs.

\subsection{Flux Relationship}

Figure 7 shows the plots of the continuum source peak flux and the integrated flux as a function of maser peak flux. Within our sample, we found no obvious relationship between the flux of methanol masers and continuum sources in both group A and group B. Thus we conclude that methanol maser luminosity is independent from the luminosity of the associated radio continuum source.

\section{SUMMARY}

We report a survey toward a large sample of Galactic class II $6.7 \mathrm{GHz}$ methanol masers and their C-band radio continuum counterparts. With the VLA in its C-configuration, we observed 372 targets selected from a variety of publications and produced a catalog of 367 methanol masers with sub-arcsecond level positional accuracy and high resolution radial velocity. We believe our catalog is a uniform and comprehensive collection of class II methanol masers in the first and second Galactic quadrants. High accuracy J2000 coordinates, radial velocities, and fluxes are presented. Maser spots detected for each source are reported in 367 individual online available files, and we plot spot maps and spectra for each maser source. We also search for compact radio continuum emission toward all masers. This resulted in detections of 279 radio continuum sources of which 157 are likely to be physically associated with a maser source. Statistical characteristics of various source properties are presented. These three catalogs may aid future investigations of massive star formation and the maser process.

This work was supported by the National Natural Science Foundation of China (Grant Number: 11133008).

\section{REFERENCES}

Argon, A. L., Reid, M. J., \& Menten, K. M. 2000, ApJS, 129, 159

Bertin, E., \& Arnouts, S. 1996, A\&AS, 117, 393

Beuther, H., Schilke, P., Sridharan, T. K., et al. 2002, A\&A, 383, 892

Brunthaler, A., Reid, M. J., Menten, K. M., et al. 2011, AN, 332, 461

Caswell, J. L. 2009, PASA, 26, 454

Caswell, J. L., \& Breen, S. L. 2010, MNRAS, 407, 2599

Caswell, J. L., Vaile, R. A., Ellingsen, S. P., Whiteoak, J. B., \& Norris, R. P. 1995, MNRAS, 272, 96

Churchwell, E. 2002, ARA\&A, 40, 27

Contreras, Y., Schuller, F., Urquhart, J. S., et al. 2013, A\&A, 549, A45

Cragg, D. M., Sobolev, A. M., \& Godfrey, P. D. 2005, MNRAS, 360, 533

Cyganowski, C. J., Whitney, B. A., Holden, E., et al. 2008, AJ, 136, 2391

Davies, B., Hoare, M. G., Lumsden, S. L., et al. 2011, MNRAS, 416, 972

Farmer, H. F. 2014, IJAA, 4, 571

Green, J. A., Caswell, J. L., Fuller, G. A., et al. 2009, MNRAS, 392, 783

Green, J. A., Caswell, J. L., Fuller, G. A., et al. 2010, MNRAS, 409, 913

Green, J. A., Caswell, J. L., Fuller, G. A., et al. 2012, MNRAS, 420, 3108

Hoare, M. G. 2005, in Ultra-Compact H II Regions (Berlin: Springer), 203

Immer, K., Brunthaler, A., Reid, M. J., et al. 2011, ApJS, 194, 25

Kauffmann, J., \& Pillai, T. 2010, ApJL, 723, L7

Kennicutt, R. C. 2005, in Proc. IAU Symp. 227, Massive Star Birth: A Crossroads of Astrophysics, ed. R. Cesaroni et al. (Cambridge: Cambridge Univ. Press), 3

Ma, C. 1997, BAAS, 29, 1236

Menten, K. M. 1991, ApJL, 380, L75

Menten, K. M., Reid, M. J., Moran, J. M., et al. 1988, ApJL, 333, L83

Minier, V., Conway, J. E., \& Booth, R. S. 2001, A\&A, 369, 278

Minier, V., Ellingsen, S. P., Norris, R. P., \& Booth, R. S. 2003, A\&A, 403, 1095

Mottram, J. C., Hoare, M. G., Davies, B., et al. 2011, ApJL, 730, L33

Pandian, J. D., Goldsmith, P. F., \& Deshpande, A. A. 2007, ApJ, 656, 255

Pandian, J. D., Momjian, E., Xu, Y., Menten, K. M., \& Goldsmith, P. F. 2011, ApJ, 730, 55

Purcell, C. R., Hoare, M. G., Cotton, W. D., et al. 2013, ApJS, 205, 1

Reid, M. J., Dame, T. M., Menten, K. M., \& Brunthaler, A. 2016, ApJ, 823, 77

Reid, M. J., Menten, K. M., Brunthaler, A., et al. 2014, ApJ, 783, 130

Reid, M. J., Schneps, M. H., Moran, J. M., et al. 1988, ApJ, 330, 809

Sanna, A., Moscadelli, L., Cesaroni, R., et al. 2010, A\&A, 517, A78

Sobolev, A. M., \& Deguchi, S. 1994, A\&A, 291, 569

Sun, Y., Xu, Y., Chen, X., et al. 2014, A\&A, 563, A130

Szymczak, M., Wolak, P., Bartkiewicz, A., \& Borkowski, K. M. 2012, AN, 333,634

Thompson, M. A., Hatchell, J., Walsh, A. J., MacDonald, G. H., \& Millar, T. J. 2006, A\&A, 453, 1003

Urquhart, J. S., Hoare, M. G., Purcell, C. R., et al. 2009, A\&A, 501, 539

Urquhart, J. S., Moore, T. J. T., Menten, K. M., et al. 2015, MNRAS, 446, 3461

Urquhart, J. S., Thompson, M. A., Moore, T. J. T., et al. 2013, MNRAS, 435,400

Walsh, A. J., Burton, M. G., Hyland, A. R., \& Robinson, G. 1998, MNRAS, 301,640

Walsh, A. J., Hyland, A. R., Robinson, G., \& Burton, M. G. 1997, MNRAS, 291, 261

Xu, Y., Li, J. J., Hachisuka, K., et al. 2008, A\&A, 485, 729

Xu, Y., Voronkov, M. A., Pandian, J. D., et al. 2009, A\&A, 507, 1117

Zinnecker, H., \& Yorke, H. W. 2007, ARA\&A, 45, 481 